\title{
XANES and EXAFS study of the local order in nanocrystalline yttria-stabilized zirconia
}

\author{
O. J. Dura, ${ }^{1,{ }^{*}}$ R. Boada, ${ }^{2, \dagger}$ M. A. López de la Torre,${ }^{1}$ G. Aquilanti, ${ }^{3}$ A. Rivera-Calzada ${ }^{4}$ C. Leon, ${ }^{4}$ and J. Chaboy ${ }^{2,5}$ \\ ${ }^{1}$ Departamento de Física Aplicada and INEI, Universidad de Castilla-La Mancha, 13071 Ciudad Real, Spain \\ ${ }^{2}$ Instituto de Ciencia de Materiales de Aragón, Consejo Superior de Investigaciones Científicas, Universidad de Zaragoza, \\ 50009 Zaragoza, Spain \\ ${ }^{3}$ Elettra-Sincrottrone Trieste, S.S. 14 km 163.534149 Basovizza, Trieste, Italy \\ ${ }^{4}$ GFMC, Departamento de Física Aplicada III, Facultad de Física, Universidad Complutense de Madrid, Campus Moncloa, \\ 28040 Madrid, Spain \\ ${ }^{5}$ Departamento de Física de la Materia Condensada, Universidad de Zaragoza, 50009 Zaragoza, Spain
}

(Received 31 January 2013; published 22 May 2013)

\begin{abstract}
The local order around $\mathrm{Zr}$ and $\mathrm{Y}$ atoms of nanocrystalline yttria-stabilized zirconia (YSZ) powders with different grain sizes has been investigated by x-ray absorption spectroscopies. The samples were prepared by means of mechanical alloying with or without subsequent sintering treatment and also by milling commercial YSZ. Our study is motivated by the interest in the electrical properties of grain boundaries and the controversy about the level of disorder in the intergrain regions in nanocrystalline YSZ. The x-ray absorption near edge structure (XANES) analysis indicates that the local order of all the sintered samples is independent of the grain size. This is confirmed by the analysis of the extended x-ray absorption fine structure, which points out also that, in contrast to that found in sintered samples, the local order around the cation in the samples milled without further sintering treatment extends only to the first coordination shell. Finally, the results of ab initio $\mathrm{Zr} K$-edge XANES calculations lead us to conclude that the observed changes of the shape of the white line are not related to a phase transformation but reflects the short-range order present in the as-milled samples.
\end{abstract}

DOI: 10.1103/PhysRevB.87.174109

PACS number(s): 81.07.Bc, 61.05.cj

\section{INTRODUCTION}

Yttria-stabilized zirconia [YSZ: $\left(\mathrm{Y}_{2} \mathrm{O}_{3}\right)_{x}\left(\mathrm{ZrO}_{2}\right)_{1-x}$ ] is a well known oxygen ion conductor. Doping with $\mathrm{Y}_{2} \mathrm{O}_{3}$ stabilizes the cubic fluorite structure of $\mathrm{ZrO}_{2}$ and supplies the oxygen vacancies responsible for the ionic conduction. ${ }^{1}$ Long-range transport of oxygen ions occurs by thermally activated hopping to adjacent oxygen vacancies, resulting in a dc conductivity, which follows an Arrhenius law. By means of chemical substitution the operating temperature of oxide-ion conductors has been reduced to the range of $700^{\circ} \mathrm{C}^{2,3}$ but this temperature is still too high for many technological applications. The effect of the dimensionality has been related with the ionic transport and, recently, nanoionics has emerged as a promising path toward enhanced values of the ionic conductivity in nanostructured materials. ${ }^{4}$ Furthermore, interesting effects appear when the characteristic dimensions of the system become comparable to the extension of the space-charge regions at grain boundaries. ${ }^{5}$

The appearance of these space-charge effects in YSZ and its consequences over the oxygen ion conductivity in nanobulk YSZ or thin films has triggered a strong controversy during the last years. ${ }^{6-9}$ While some authors reported an enhancement in the ionic conductivity in samples with nanometer size grains, ${ }^{10}$ others have found no changes ${ }^{11}$ or even a decrease of the ionic conductivity $^{12}$ in samples obtained with different synthesis methods, with different grain sizes, and even with different dopant concentrations.

In this scenario investigating the microstructural and compositional differences between the grain boundary and the average (bulk) atomic order, would help to clarify the role played by grain boundaries on the macroscopic transport properties of YSZ. In order to address this issue we report a deep x-ray absorption spectroscopy (XAS) analysis of the
YSZ samples, including both the X-ray absorption near edge structure (XANES) and extended $\mathrm{x}$-ray absorption fine structure (EXAFS). Herein we focus on studying the occurrence of size effects through the determination of the local structure around $\mathrm{Zr}$ and $\mathrm{Y}$ cations of nanocrystalline $10 \%$ mol YSZ samples, $\left(\mathrm{Y}_{2} \mathrm{O}_{3}\right)_{0.1}\left(\mathrm{ZrO}_{2}\right)_{0.9}$, obtained by mechanical alloying (MA) with controlled grain sizes obtained by sintering at different temperatures. In addition, in order to exclude any effect from the mechanical alloying method used for the sample preparation, we have extended the study to samples prepared from commercial YSZ powders.

In a previous work an exhaustive electrical characterization of the $10 \mathrm{~mol} \%$ YSZ mechanically alloyed samples was made by using impedance spectroscopy. ${ }^{12}$ It was reported that the conductivity associated to oxide ions traveling through the grain-boundary core from one grain to another one is similar for samples with grain sizes ranging from $\sim 1 \mu \mathrm{m}$ to $17 \mathrm{~nm}$, i.e., it is rather independent of the grain size (see Fig. 1, right axis). In contrast the bulk conductivity associated to oxide ions diffusing inside the grains decreases more than one order of magnitude when the grain size decreases (Fig. 1, left axis). These results were interpreted in terms of the formation of a space-charge layer whose thickness increases up to $7 \mathrm{~nm}$ when the grain size is reduced to the nanometer range. ${ }^{12}$

\section{EXPERIMENT AND COMPUTATION METHODS}

Nanocrystalline $10 \%$ mol YSZ powders were obtained by mechanical alloying a stoichiometric mixture of $\mathrm{ZrO}_{2}$ and $\mathrm{Y}_{2} \mathrm{O}_{3}$, both commercial powders purchased from Alfa Aesar. The process was carried out in a Retsch PM400 planetary ball mill with YSZ pots and balls. The alloying process was monitored using $\mathrm{x}$-ray diffraction measurements 


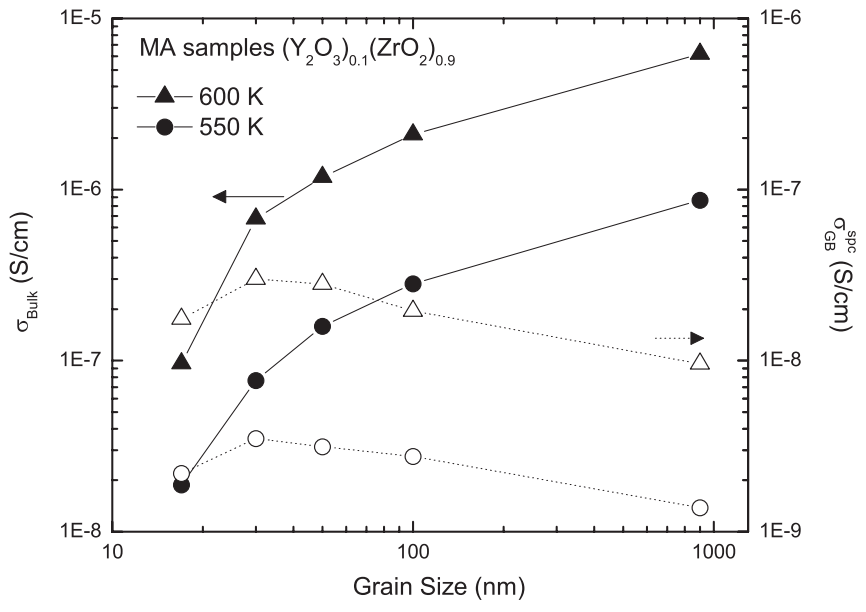

FIG. 1. (Left axis, solid symbols) Bulk ionic conductivity as a function of grain size for samples obtained by mechanical alloying at $600 \mathrm{~K}$ (triangles) and at $550 \mathrm{~K}$ (circles). (Right axis, open symbols) Specific grain boundary ionic conductivity for the same samples at the same temperatures.

at different milling times. The yttria concentration of the asmilled samples was determined from x-ray energy dispersive spectroscopy measurements. Powders obtained after alloying for $48 \mathrm{~h}$ were pressed into compacts. These compacts were sintered at temperatures between $750{ }^{\circ} \mathrm{C}$ and $1350{ }^{\circ} \mathrm{C}$ to obtain samples with different grain size. Grain-size values of these samples were determined from a Warren-Averbach line profile analysis of the XRD patterns. Complementary AFM experiments were also performed in selected samples in order to obtain an independent estimate of the grain size and compare it with the values obtained from XRD. In all cases a good agreement was found between both techniques. The values for the nominal average sizes of the grains as obtained from the XRD data are summarized in Table I. More details about sample preparation and characterization were reported in previous works. ${ }^{12,13}$ The mechanically alloyed sample is labeled as MA, and sintered samples as MA-1350,
MA-950, MA-850, and MA-750, where the number indicates the sintering temperature. In addition we have also studied commercial powders of $10 \% \mathrm{~mol}$ YSZ (Tosoh-Europe), $5.4 \%$ mol YSZ (Alfa Aesar), and $\mathrm{ZrO}_{2}$ (Alfa Aesar), hereafter labeled C-10, C-5, and C-Zr respectively. These commercial powders were mechanically milled (MM) to reduce the grain size and therefore obtain samples with different degrees of order/disorder. These samples were labeled as CMM-10, CMM-5, and CMM-Zr respectively.

XAS spectra were obtained at the bending magnet XAFS beamline at the ELETTRA Synchrotron (Trieste, Italy). ${ }^{14}$ Experiments were performed at room temperature in transmission mode with ionization chambers (Oxford Instruments) filled with optimal gas mixtures of $\mathrm{He}, \mathrm{N}_{2}, \mathrm{Ar}$, and $\mathrm{Kr}$. The energy of the incoming $\mathrm{X}$-ray beam was tuned with a $\mathrm{Si}(111)$ double-crystal monochromator in the energy range from 16 to $20 \mathrm{keV}$ covering the $\mathrm{Y}$ and $\mathrm{Zr} K$ edge. This setup provides an energy resolution $\Delta E / E \sim 10^{-4}$. For the XAS measurements homogeneous layers of powdered samples were prepared by spreading fine powders of the material onto an adhesive tape. Thickness and homogeneity of the samples were optimized to obtain the best signal-to-noise ratio. The XAS spectra were analyzed according to standard procedures ${ }^{15}$ using the ATHENA program pack. ${ }^{16}$

The quantitative EXAFS analysis was carried out using the phase and amplitude transferability method. ${ }^{17,18}$ EXAFS data fitting were performed by using the VIPER program. ${ }^{19}$ In all cases the backscattering amplitude, phase and photoelectron mean free path factor were obtained from the experimental EXAFS spectra of the appropriate reference compounds. For this comparative study, the Fourier transforms of the EXAFS signals were performed within the same $k$ range. The ab initio computation of the XANES spectra was carried out by using the multiple-scattering code CONTINUUM, ${ }^{20,21}$ included in the MXAN program pack. ${ }^{22}$ A complete discussion of the procedure can be found elsewhere. ${ }^{23-25}$ It should be stressed that no free parameters have been used during the calculations. The theoretically calculated spectra have been directly compared to the experimental XANES spectrum, i.e., no fitting procedure

TABLE I. Volume-averaged grain size, yttrium content, and complementary information about the samples studied in this work.

\begin{tabular}{|c|c|c|c|}
\hline $\begin{array}{l}\text { Sample } \\
\text { label }\end{array}$ & Grain Size & $\begin{array}{c}\mathrm{Y}_{2} \mathrm{O}_{3} \text { Concentration } \\
(\mathrm{mol} \%)\end{array}$ & Remarks \\
\hline $\mathrm{C}-10$ & Micron size & 10 & Commercial reference powder \\
\hline MA-1350 & Micron size & 10 & Mechanically alloyed and subsequent sintering \\
\hline MA-950 & $100 \mathrm{~nm}$ & 10 & Mechanically alloyed and subsequent sintering \\
\hline MA-850 & $50 \mathrm{~nm}$ & 10 & Mechanically alloyed and subsequent sintering \\
\hline MA-750 & $30 \mathrm{~nm}$ & 10 & Mechanically alloyed and subsequent sintering \\
\hline MA & $17 \mathrm{~nm}$ & 10 & Mechanically alloyed (48 h.) \\
\hline MA372h & $17 \mathrm{~nm}$ & 10 & Mechanically alloyed (372 h.) \\
\hline CMM-10 & $21 \mathrm{~nm}$ & 10 & Commercial powder milled ( $48 \mathrm{~h})$. \\
\hline C-5 & Micron size & 5 & Commercial reference powder \\
\hline CMM-5 & $16 \mathrm{~nm}$ & 5 & Commercial powder milled (48 h.) \\
\hline $\mathrm{C}-\mathrm{Zr}$ & Micron size & 0 & Commercial reference powder \\
\hline $\mathrm{CMM}-\mathrm{Zr}$ & $16 \mathrm{~nm}$ & 0 & Commercial powder milled (48 h.) \\
\hline $\mathrm{Y}_{2} \mathrm{O}_{3}$-Ref & Micron size & 100 & Commercial reference powder \\
\hline
\end{tabular}


has been used. The assessment of the quality of the theoretical computations is based on the correct reproduction of the shape and energy position of the different spectral features and of their relative energy separation and the intensity ratio. In all the cases, the theoretical spectra have been convoluted with a Lorentzian shape function to account for the core-hole lifetime ${ }^{26}$ and the experimental resolution.

\section{RESULTS AND DISCUSSION}

In view of the oxide-ion conductivity introduced above it is important to look for any structural changes in the bulk or the grain boundary region that might affect the conductivity values. Aiming to investigate any differences between the grain boundary and the bulk we have performed a detailed XANES and EXAFS study at the $\mathrm{Zr}$ and $\mathrm{Y} K$ edge. The short-range character of these techniques will allow us to determine the local order around the cation sites (both $\mathrm{Zr}$ and $\mathrm{Y}$ ) in the samples and to monitor its dependence on the sintering temperature, i.e., on the grain size. Several XAS studies have been reported to date for solid $\mathrm{ZrO}_{2}-\mathrm{Y}_{2} \mathrm{O}_{3}$ solutions or nanocrystalline YSZ materials for which the structural disorder plays an important role. ${ }^{27,28}$ The results were controversial, mainly due to the interpretation of the coordination numbers and Debye-Waller factors extracted from EXAFS. ${ }^{29}$ For this reason not only the EXAFS spectra but also the XANES region, more sensitive to the bonding geometry and stereochemical details, have been analyzed in order to reduce the possible arbitrariness of the EXAFS results arising from the large uncertainties in coordination numbers and Debye-Waller factors due to their strong correlation.

\section{A. XANES: qualitative analysis}

The comparison of the XANES spectra recorded at the $\mathrm{Zr}$ $K$ edge in all the studied samples is shown in Fig. 2. First, we compare in Fig. 2(a) the spectra of the commercial $10 \%$ mol YSZ sample (C-10) used as reference, and those of samples obtained by mechanical alloying (MA) and subsequent sintering (MA-1350, MA-950, MA-850, and MA-750). For the sake of completeness the XANES spectra of the MA sample without sintering but further milled (MA372h) is also included.

All the sintered MA samples show a similar spectral shape as for the $\mathrm{C}-10$ reference. In all these cases the $\mathrm{Zr} K$ edge XANES is characterized by a main peak or white line split in two components, $\mathrm{A}$ and $\mathrm{B}$, at $\sim 6 \mathrm{eV}$ and $12 \mathrm{eV}$ above the absorption edge, respectively (the absorption edge was defined as the maximum of the first derivative of the C-10 XANES spectrum, $E=18010 \mathrm{eV}$ ). In addition, a small rounded peak (C) and a broad resonance (D) are found, respectively, at $E-$ $E_{0} \sim 34 \mathrm{eV}(\mathrm{C})$ and $67 \mathrm{eV}(\mathrm{D})$. The B/A intensity ratio of all the sintered samples is similar to that of the $\mathrm{C}-10$ bulk reference with the exception of MA-750 for which feature B is less marked. In contrast, the characteristic double-peak white line is lost in the case of the parent MA sample for which also the $\mathrm{C}$ peak at higher energies has disappeared. Interestingly, the XANES spectra of both MA and MA372h samples are closely similar. Thus, the XANES spectra are independent of the milling time.
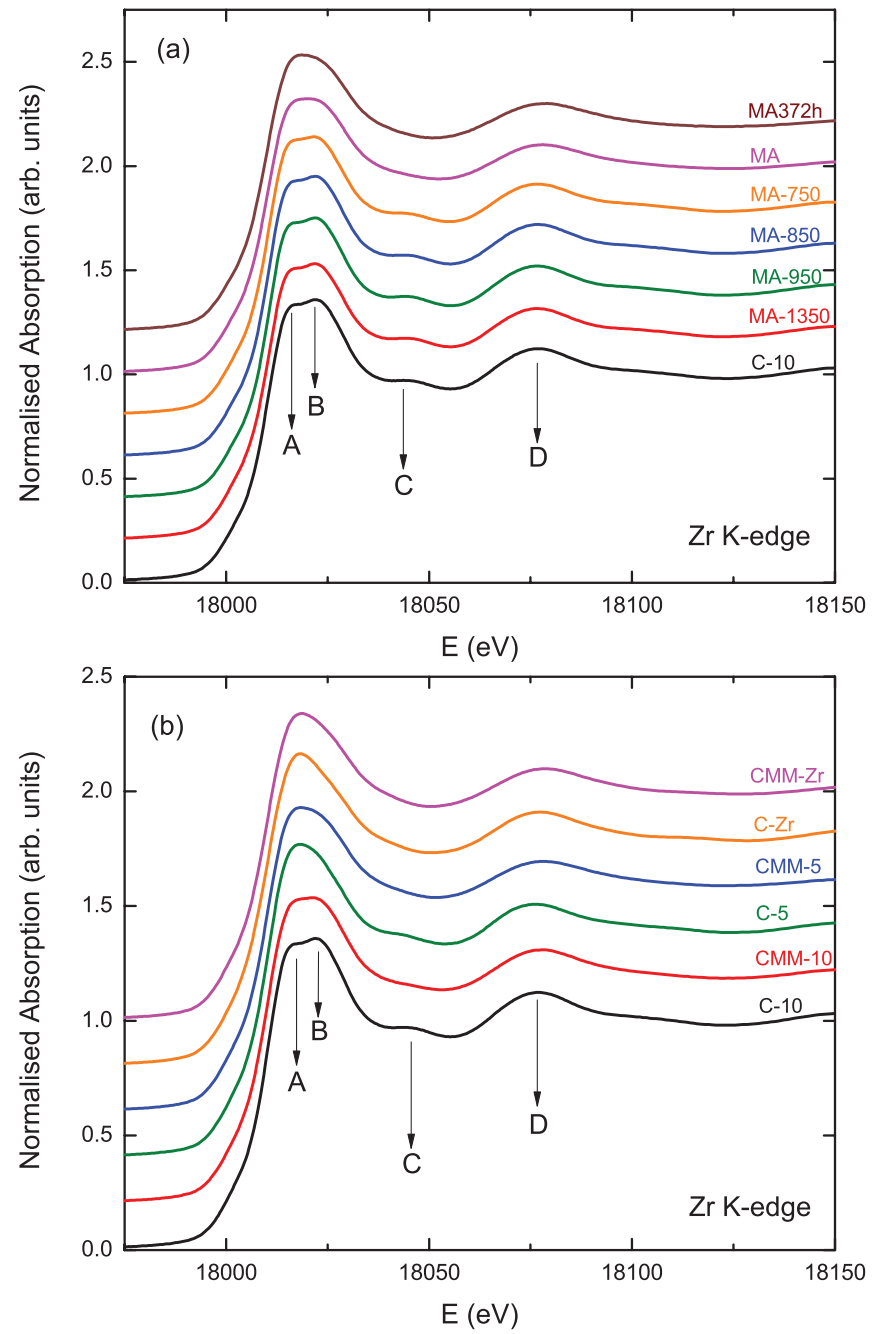

FIG. 2. (Color online) Comparison between the normalized $\mathrm{Zr}$ $K$-edge XANES spectra of the nanocrystalline samples obtained by mechanical alloying (top panel) and milling (bottom panel) with the reference commercial powders. Spectra have been vertically shifted for the sake of clarity.

This fingerprint analysis indicates that all the sintered samples show the same local order around $\mathrm{Zr}$ sites, identical to that observed in the $\mathrm{C}-10$ reference, i.e., the observed local order is independent of the grain size which is determined by the sintering temperature. Indeed, samples mechanically alloyed but without further sintering (both MA and MA372h) exhibit less local order than the sintered or commercial ones as determined from XANES. The XANES spectra is nearly identical for both samples and clearly different from that of the sintered samples, indicating higher structural disorder. This last result is in agreement with the behavior observed in the comparison of the XANES spectra of all the commercial reference samples prior and after milling without sintering process [see Fig. 2(b)]. Moreover, the $\mathrm{Zr} K$-edge XANES spectra show characteristic features, marked with arrows in Fig. 3, depending on the structural phase. Accordingly, the spectra observed for the C-10 reference and sintered MA samples correspond to a cubic phase: in addition to the lack of the pre-edge structure observed for the tetragonal phase, 


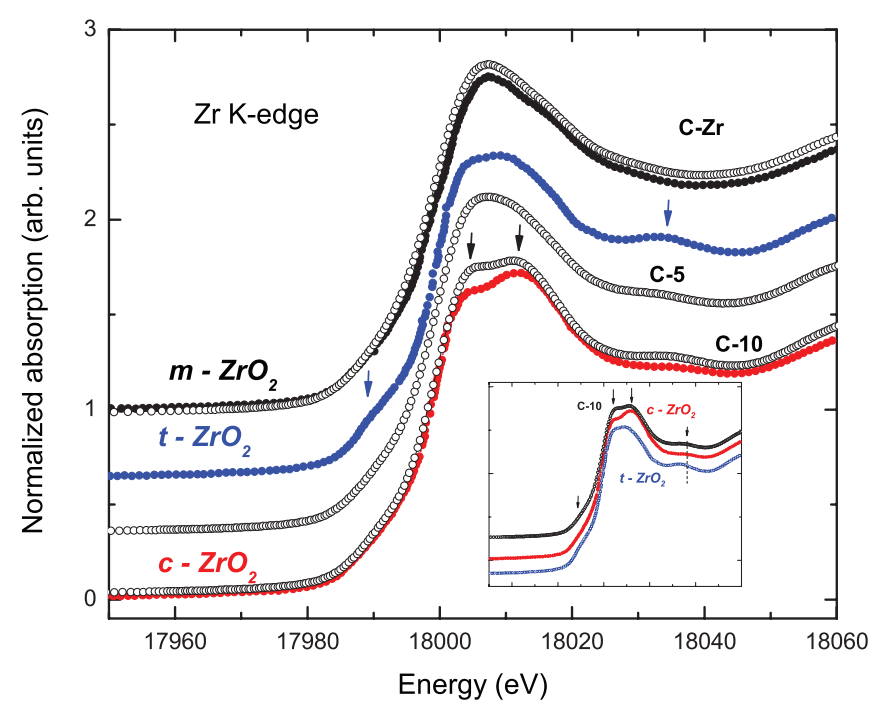

FIG. 3. (Color online) Comparison between the normalized $\mathrm{Zr}$ $K$-edge XANES spectra of the $\mathrm{ZrO}_{2}, 5 \% \mathrm{YSZ}$ and $10 \% \mathrm{YSZ}$ reference samples (this study) and those of zirconia polymorphs (from Ref. 30). Spectra have been vertically shifted for the sake of clarity. In the inset a detailed comparison of the spectra of $10 \%$ YSZ sample and those of cubic and tetragonal zirconia are shown.

the shape and the relative intensity of the two-peaks structure of the main absorption line, as well as the energy position of the small bump appearing at higher energies resemble those of cubic zirconia. On the contrary, the situation is less clear in the case of the 5\% YSZ sample and the presence of a tetragonal phase cannot be discarded from this fingerprint analysis. The same holds for all the milled samples for which no sintering was applied. This issue will deserve a detailed discussion in connection with the $a b$ initio XANES computations discussed later.

Similar results are found at the Y $K$ edge, i.e., when the local order around the $\mathrm{Y}$ site is being probed (see Fig. 4). In the case of the C-10 reference and of the sintered MA samples the $\mathrm{Y} K$-edge XANES is characterized by an asymmetric white line with a main peak at $E-E_{0} \sim 4.5 \mathrm{eV}$ and a high-energy shoulder $\mathrm{B}$ at about $11.5 \mathrm{eV}$ above the edge (the zero of the energy scale is defined as the maximum of the first derivative of the C-10 XANES spectrum, $\left.E_{0}=17048 \mathrm{eV}\right)$. Just after the white line a double step resonance is found $(C \sim 39 \mathrm{eV}$; $D \sim 60 \mathrm{eV}$ ), and for higher energies two additional structures ( $E \sim 100 \mathrm{eV} ; F \sim 135 \mathrm{eV}$ ). The characteristic energies of the different spectral features differ from those of $\mathrm{Y}_{2} \mathrm{O}_{3}$ which indicates that the local structure around $\mathrm{Y}$ in the different YSZ materials is not the same as for the $\mathrm{Y}_{2} \mathrm{O}_{3}$ reference. Moreover, the spectra of the sintered samples show peaked resonances characteristic of a well defined local order. As in the case of the $\mathrm{Zr} K$ edge, all the sintered samples and the C-10 reference exhibit similar spectra independently of the grain size. In contrast, the spectra of the mechanically alloyed samples (both MA and MA372h) show broadened resonances and, in particular, the B component of the white line or the $E$ and $F$ peaks at high energy become not detectable.

XANES results, both at the $\mathrm{Zr}$ and $\mathrm{Y} K$ edge, indicate the existence of a well defined local order around the cation sites in the YSZ samples obtained by mechanical alloying
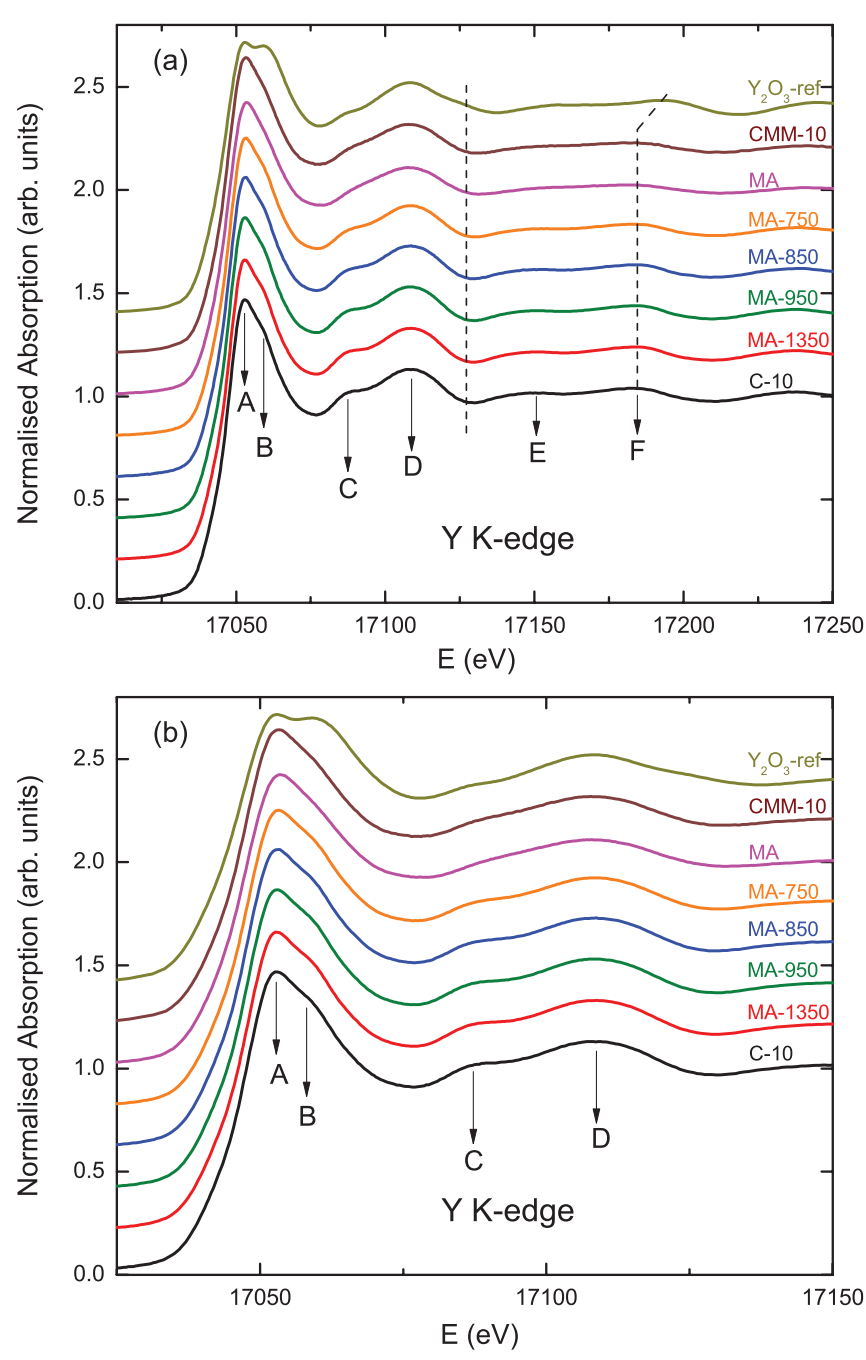

FIG. 4. (Color online) Top: Comparison between the normalized Y $K$-edge XANES spectra of the nanocrystalline samples obtained by mechanical alloying and milling and reference commercial powders. Spectra have been vertically shifted for the sake of clarity. Bottom: The same data are displayed in a narrower range.

followed by a sintering treatment at high temperatures. This local order does not depend of the grain size, controlled through the sintering temperature (see Table I), being similar for commercial and mechanical alloyed samples. However, mechanically alloyed samples for which no sintering was performed show a less ordered environment around both $\mathrm{Zr}$ and Y (see comparison of MA and MA372h samples in Fig. 2 and Fig. 4).

\section{B. EXAFS analysis}

A deeper knowledge of the local order around both $\mathrm{Zr}$ and $Y$ cations can be obtained from the quantitative analysis of the EXAFS spectra. The comparison of the $\mathrm{Zr} K$-edge $\mathrm{k}^{3}$-weighted EXAFS signal and their Fourier transforms (FTs) are shown in Fig. 5. The EXAFS signals, $\chi(k)$, have been obtained after removing the background by a cubic spline polynomial fitting and by normalizing the magnitude of the oscillations to the edge jump. The corresponding pseudoradial distribution function around the absorbing atom has been obtained by 

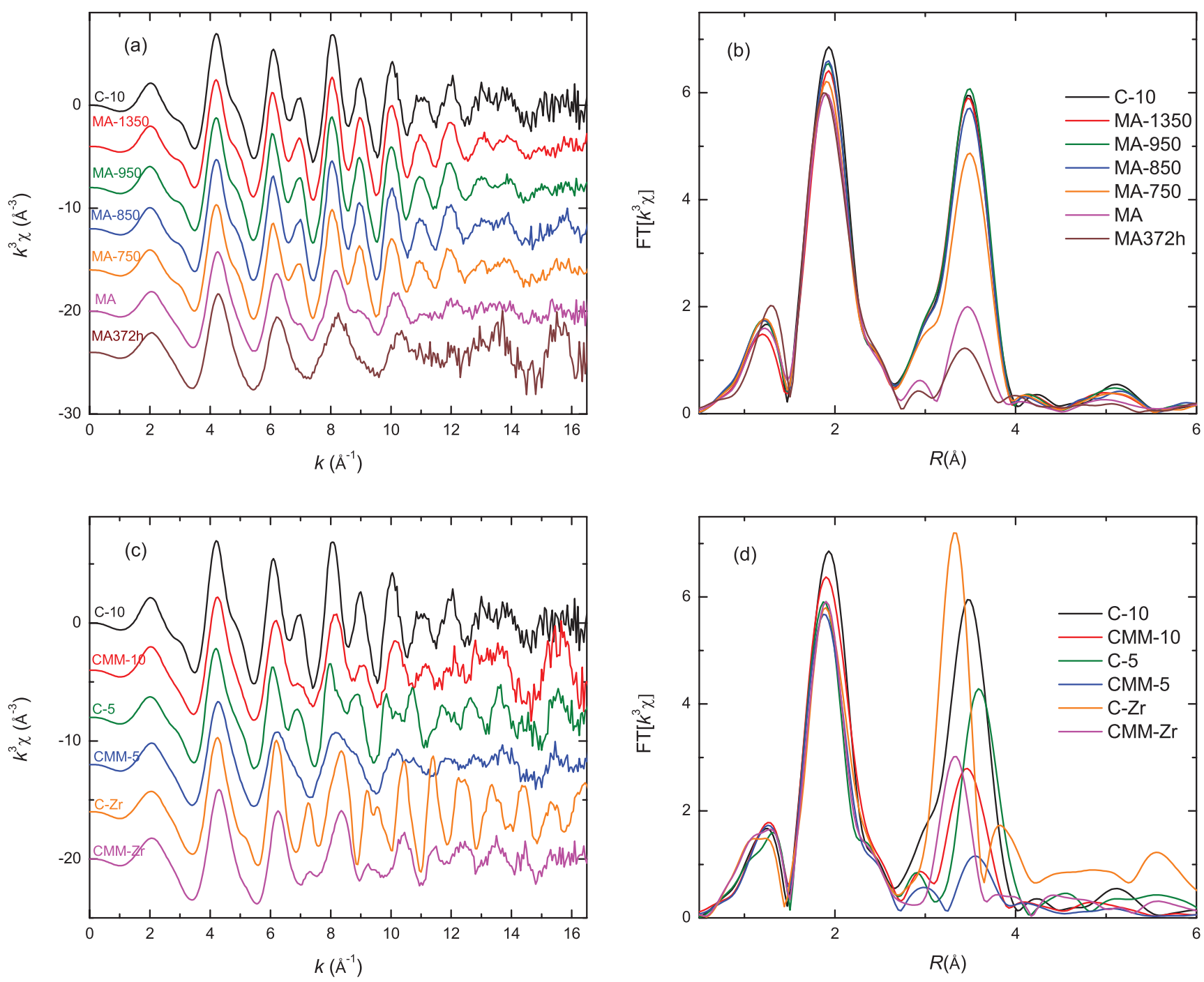

FIG. 5. (Color online) Comparison between the experimental Zr $K$-edge $k^{3} \chi$ EXAFS signal (left) and the modulus of the Fourier transforms (right) in the mechanical alloyed, milled, and commercial samples.

performing the Fourier transform (using a gaussian window) in the range $1.5 \leqslant k \leqslant 12 \AA$.

As shown in the figure, the EXAFS signals are similar for the commercial reference C-10 and all the sintered MA samples, whereas the spectra relative to the MA and MA372h present different and more broader features, in agreement with the XANES results. However, the inspection of the FTs shows that the contribution of the second coordination shell of the sample sintered at $750^{\circ} \mathrm{C}$ is significantly smaller than for the rest of the samples within the series. This result indicates that, despite the local order around $\mathrm{Zr}$ is the same in all the cases this particular sample is more disordered, i.e., only the nearest neighbors show a well defined local ordering. It is necessary to raise the sintering temperature to extent the local order to further coordination shells. This behavior is similar to that observed for the samples obtained by alloying without sintering. In all these cases the intensity of the second peak in the FT is much smaller than for the sintered ones.

Several authors have reported the strong attenuation of the EXAFS for similar ball-milled samples in comparison to that for their bulk parents. ${ }^{27}$ This effect is commonly interpreted as the ball-milled sample containing a large level of disorder or as it is due to the presence of amorphous material in the sample. ${ }^{31}$ The reduction of the amplitude of the second peak in the Fourier transform, which is due to the $\mathrm{Zr}-\mathrm{Zr}$ correlation, is a common finding in EXAFS studies of nanocrystalline materials. This feature is often interpreted as evidence of high levels of disorder in nanocrystalline materials. However, as stated by Chadwick et al. ${ }^{27}$ it is not possible to identify the exact nature of disorder on the basis of the EXAFS experiment alone. Possible origins of this disorder include: (i) the presence of a large number of defects and ill-coordinated surface atoms; (ii) highly disordered interfaces between crystallites, and (iii) the existence of an amorphous phase. The intergrain regions could also be amorphous in nature, which would clearly produce an attenuation of the EXAFS signal and a reduction of the peak amplitudes in the Fourier transform. However, the qualitative effect in EXAFS is the same as that produced by the simple reduction of crystallite size, and care has to be taken over the sample preparation and measurement procedures 
as on the EXAFS analysis to separate the two effects, ${ }^{29,32}$ as illustrated in the current controversy over the level of disorder in the intergrain regions in the case of nanocrystalline metals. ${ }^{32-35}$

In this respect, Qi et al. reported in their EXAFS study of nanocrystalline YSZ that the cation-cation $(\mathrm{Zr}-\mathrm{Zr})$ coordination number is reduced due to the large number of cations near the surface (with fewer nearest neighbors) when the grain size decreases. ${ }^{36}$ This is in contrast with the conclusions reached by Rush et al. on nanocrystalline YSZ films. ${ }^{37}$ They found that the $\mathrm{Zr}$ and $\mathrm{Y} K$ edge EXAFS spectra for the YSZ films with grain sizes of 6, 15, and $240 \mathrm{~nm}$ showed no major differences with the corresponding spectra of the bulk counterpart. This is clear proof that these nanocrystalline films exhibit similar levels of disorder to that of large crystals. Consequently, there is no support for the view that the intergrain regions are highly disordered. The results of Rush et al. are in agreement with our findings, showing the similarity of the structural order at the grain boundaries independently of the decreasing grain size. If the structure of the grain boundaries is quite different from that of the bulk, one should expect also markedly different electrical properties. If this is the case, our finding of a nearly constant grain boundary conductivity should be affected and our estimates of the bulk conductivity too. However, as shown in Fig. 5, all samples display the same spectral shape showing a perfect coincidence in the energy position and relative intensities of the different EXAFS features. It is important to note that the FT intensities are similar for all the sintered samples, whose grain sizes are in the range from $1 \mu \mathrm{m}$ to $\sim 30 \mathrm{~nm}$. If the grain boundaries were more disordered than the bulk their effect should become more important into the EXAFS for the samples with smaller grain size, contrary to the present results. Moreover, the EXAFS results indicate that the local order at the first coordination shell is similar for all the studied compounds, i.e., for either milling or alloying process. In contrast, only sintered samples exhibit well defined second coordination shells indicating that mechanically alloying without sintering yields highly disordered compounds, in agreement with the observed strong depletion of their bulk conductivity.

In order to get a quantitative estimate of the aforesaid modifications we have performed simulations of the EXAFS spectra by using the phase transferability method and the VIPER program. Backscattering amplitudes and phases were obtained from the experimental EXAFS spectra of the $\mathrm{C}-10$ reference compound. In this way the only parameters allowed to vary during the fitting procedure were the $\mathrm{Zr}-\mathrm{O}$ and $\mathrm{Zr}-\mathrm{Zr}(\mathrm{Y})$ interatomic distances $\left(r_{\mathrm{Zr}-\mathrm{O}}, r_{\mathrm{Zr}-\mathrm{Zr}}\right)$ and the differential Debye-Waller factor, $\Delta \sigma^{2}$, relative to that of the $\mathrm{C}-10$ reference sample. The EXAFS fitting has been performed for the contribution of the first and second coordination shells separately, and also by including both coordination shells in a single fitting. For this purpose the EXAFS contribution of these coordination shells was isolated by Fourier filtering: both contributions were back-transformed into $k$ space in the $1.13 \leqslant R \leqslant 2.04 \AA$ and $2.77 \leqslant R \leqslant 3.62 \AA$ ranges, respectively. Then, the difference of the local environment of $\mathrm{Zr}$ in the studied samples was assessed by analyzing the filtered EXAFS signals (see Fig. 6). The fitting of the EXAFS signals were made by imposing integer coordination
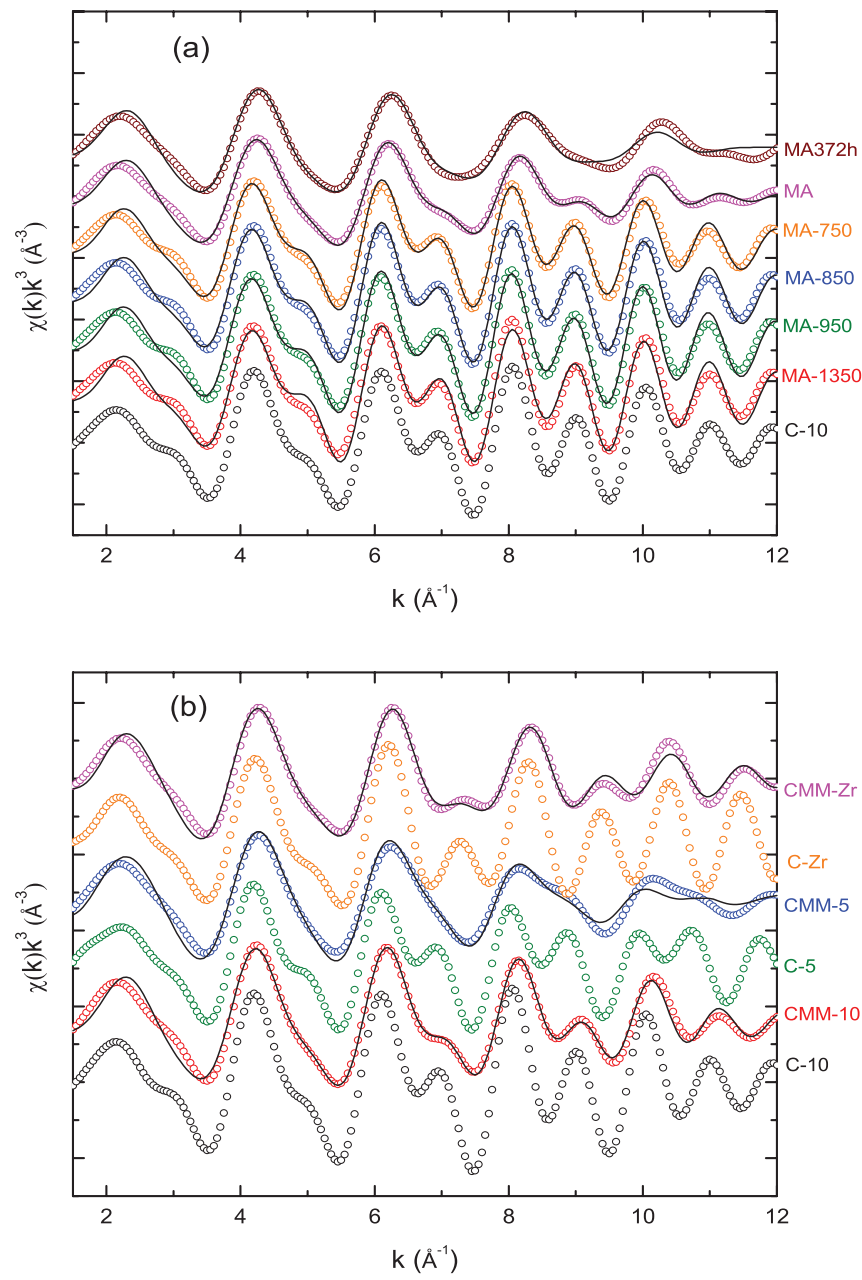

FIG. 6. (Color online) Comparison between the $\mathrm{Zr} K$-edge back Fourier transform (BFT) of the nanocrystalline samples obtained by mechanical alloying (top) and milling (bottom). Solid lines represent the fits corresponding to the parameters displayed in Table II.

number, $N_{\text {Coord }}$, values; fixing the interatomic distances equal to those of the reference and by allowing them to vary. The best-fit parameters are summarized in Table II. A similar study has been performed at the Y $K$ edge (see Fig. 8 and Table III).

The EXAFS results indicate, in agreement with XANES, that mechanical alloyed samples subject to a sintering process show a well established order around the cations. Indeed, no difference of this environment is found among the sintered samples and the reference compounds obtained by standard synthesis methods. However, a more detailed analysis indicates that the sample sintered at $750{ }^{\circ} \mathrm{C}$ shows that the second coordination shell around $\mathrm{Zr}$ is more disordered that for the rest of the sintered samples (see Fig. 5). Interestingly, in the case of $\mathrm{Y}$ this increased disorder extends also to the sample sintered at $850{ }^{\circ} \mathrm{C}$ (see Fig. 7). Despite these differences the coordination numbers determined from EXAFS are the same, within the error, in all cases. In contrast, all the samples obtained by mechanically alloying and milling without further sintering show a high disorder in the second coordination shell, i.e., a reduced coordination number $(\sim 50 \%)$ and the concomitant contraction $(\sim 0.02-0.04 \AA$ ) of the interatomic distances (see 
TABLE II. Best fit parameters obtained from the analysis of the first and second shell contributions to the Zr $K$-edge EXAFS spectra. ${ }^{38} N_{i}$ : coordination number; $r$ : distance; $\Delta \sigma^{2}$ : Debye-Waller factor, $\sigma^{2}$, relative to that of the corresponding reference sample; $\mathcal{R}:$ reliability factor. ${ }^{19}$

\begin{tabular}{lcccccc}
\hline \hline Sample & $N_{1}$ & $r_{Z r-O}(\AA)$ & $\Delta \sigma_{Z r-O}^{2}\left(\AA^{2}\right)$ & $N_{2}$ & $r_{Z r-Z r}(\AA)$ & $\Delta \sigma_{Z r-Z r}^{2}\left(\AA^{2}\right)$ \\
\hline C-10 & 8 & 2.23 & 0 & 12 & 3.64 & $\mathcal{R}$ \\
MA-1350 & 8 & $2.23 \pm 0.01$ & $0.001 \pm 0.001$ & 12 & $3.64 \pm 0.02$ & $0.000 \pm 0.001$ \\
MA-950 & 8 & $2.23 \pm 0.01$ & $0.001 \pm 0.001$ & 12 & $3.65 \pm 0.02$ & $0.000 \pm 0.001$ \\
MA-850 & 8 & $2.23 \pm 0.01$ & $0.001 \pm 0.001$ & 12 & $3.64 \pm 0.02$ & $0.000 \pm 0.001$ \\
MA-750 & 8 & $2.23 \pm 0.01$ & $0.001 \pm 0.001$ & $11 \pm 1$ & $3.65 \pm 0.02$ & $0.001 \pm 0.001$ \\
MA & 8 & $2.22 \pm 0.01$ & $0.002 \pm 0.001$ & $6 \pm 2$ & $3.60 \pm 0.02$ & $0.003 \pm 0.002$ \\
MA372h & 8 & $2.22 \pm 0.01$ & $0.002 \pm 0.001$ & $6 \pm 2$ & $3.56 \pm 0.02$ & $0.009 \pm 0.003$ \\
CMM-10 & 8 & $2.23 \pm 0.01$ & $0.001 \pm 0.001$ & $6 \pm 2$ & $3.61 \pm 0.02$ & $0.001 \pm 0.002$ \\
C-5 & 8 & 2.23 & 0 & 12 & 3.64 & 0.05 \\
CMM-5 & 8 & $2.23 \pm 0.01$ & $0.001 \pm 0.001$ & $6 \pm 2$ & $3.60 \pm 0.02$ & 0.007 \\
C-Zr & 8 & 2.17 & 0 & 12 & 3.74 & $0.006 \pm 0.003$ \\
CMM-Zr & 8 & $2.16 \pm 0.01$ & $0.001 \pm 0.001$ & $6 \pm 2$ & $3.73 \pm 0.02$ & $0.001 \pm 0.003$ \\
\hline \hline
\end{tabular}

Tables II and III). This last result indicates that the milling process leads to YSZ-like compounds with very short local order and a high degree of structural disorder. This disorder fades up upon sintering, proceeding faster around the $\mathrm{Zr}$ sites than those of the $\mathrm{Y}$.

A final discussion is devoted to the study of the local order around the cation in the milled samples. Our aim is to elucidate if the high degree of disorder induced in the process implies a modification of the structural phase departing from the original one (monoclinic or cubic) or, on the contrary, the original local order is retained. This is also a controversial issue. Several authors have concluded that there is a tetragonal to cubic transition that occurs upon reduction of the crystallite size ${ }^{39}$ for others, the parent crystalline material, monoclinic $\mathrm{ZrO}_{2}$, evolves into a nanocrystalline phase that is locally similar to monoclinic zirconia but shows a cubic-type ordering at nanometer range distances. ${ }^{40}$ In this respect the study of XANES can provide useful complementary information as XANES is extremely sensitive to the stereochemical details of the absorbing site, i.e., overall symmetry, interatomic distances, and bond angles, offering better capabilities than a direct EXAFS analysis for the determination of the coordination polyhedron around the absorbing atom. ${ }^{41-43}$

\section{C. $A b$ initio XANES calculations}

To this aim we have performed $a b$ initio $\mathrm{Zr} K$-edge XANES calculations. During the computations special attention has been paid to determine the best choice for the overlapping factor between the muffin-tin spheres and for the exchange and correlation part of the final state potential. ${ }^{44-46}$ We have found that the best reproduction of the experimental data is obtained by using an overlapping factor of $10 \%$ and the real Dirac-Hara ECP potential, in agreement to previous works. ${ }^{47,48}$ As shown in the top panel of Fig. 9 we have first verified that the computations are able of reproducing the characteristic spectral features of the different polymorphs as reported in Fig. 3. Then we have performed computations for a cubic YSZ cluster of 99 atoms, i.e., including all the contributions of atoms within the first $6.3 \AA$ around the absorbing atom. As shown in Fig. 9 the spectral shape of the experimental spectrum of the sample C-10 (in black dots) is qualitatively well reproduced. In particular, the computation shows the existence of two contributions at the main absorption structure. It should be noted that the experimental spectral shape is not obtained when the computation includes only the contribution of the first two coordination shells around photoabsorbing $\mathrm{Zr}$. Interestingly, the double-peak structure obtained for the big
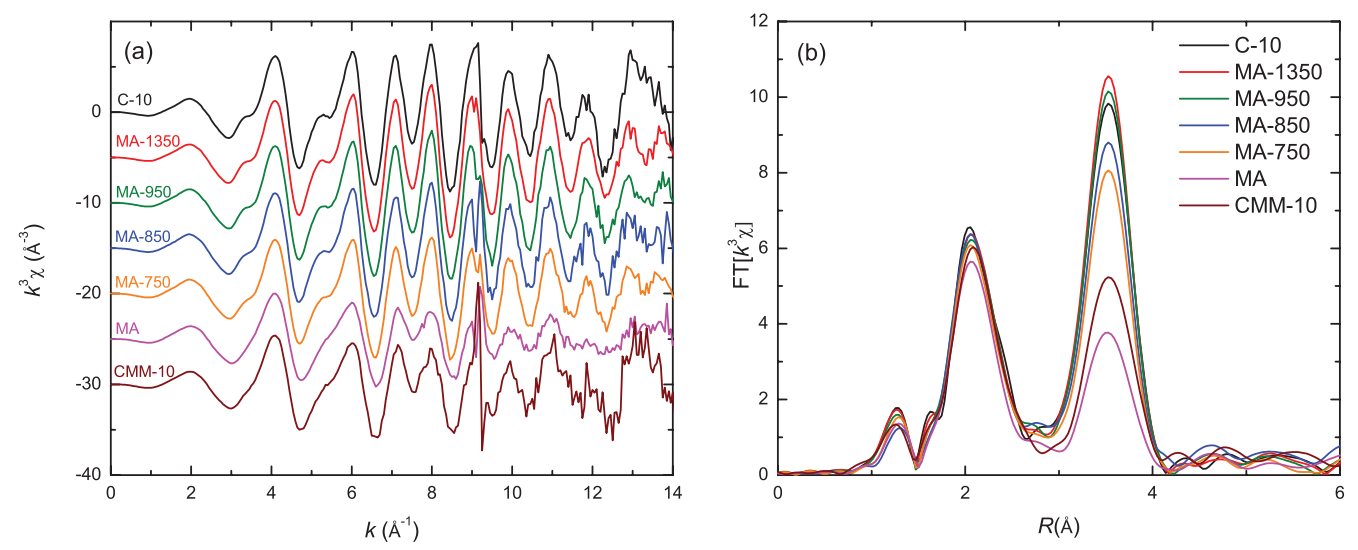

FIG. 7. (Color online) Comparison between the experimental Y $K$ edge $k^{3} \chi$ EXAFS signal (left) and the modulus of the Fourier transforms (right) of the samples studied in this work. 
TABLE III. Best fit parameters obtained from the analysis of the first and second shell contribution to the Y $K$-edge EXAFS spectra. $N_{i}$ : coordination number; $r$ : distance; $\Delta \sigma^{2}$ : Debye-Waller factor, $\sigma^{2}$, relative to that of the corresponding reference sample; $\mathcal{R}$ : reliability factor.

\begin{tabular}{lcccccc}
\hline \hline Sample & $N_{1}$ & $r_{Y-O}(\AA)$ & $\Delta \sigma_{Y-O}^{2}\left(\AA^{2}\right)$ & $N_{2}$ & $r_{Y-Z r}(\AA)$ & $\Delta \sigma_{Y-Z r}^{2}\left(\AA^{2}\right)$ \\
\hline C-10 & 8 & 2.23 & 0 & 12 & 3.64 & 0 \\
MA-1350 & 8 & $2.23 \pm 0.01$ & $0.000 \pm 0.001$ & 12 & $3.63 \pm 0.02$ & $0.000 \pm 0.001$ \\
MA-950 & 8 & $2.23 \pm 0.01$ & $0.000 \pm 0.001$ & 12 & $3.63 \pm 0.02$ & $0.000 \pm 0.001$ \\
MA-850 & 8 & $2.23 \pm 0.01$ & $0.000 \pm 0.001$ & 12 & $3.63 \pm 0.02$ & $0.000 \pm 0.001$ \\
MA-750 & 8 & $2.23 \pm 0.01$ & $0.001 \pm 0.001$ & $11 \pm 1$ & $3.62 \pm 0.02$ & $0.001 \pm 0.002$ \\
MA & 8 & $2.22 \pm 0.01$ & $0.002 \pm 0.001$ & $7 \pm 2$ & $3.61 \pm 0.02$ & $0.003 \pm 0.002$ \\
CMM-10 & 8 & $2.22 \pm 0.01$ & $0.001 \pm 0.001$ & $7 \pm 2$ & $3.62 \pm 0.02$ & $0.001 \pm 0.002$ \\
\hline \hline
\end{tabular}

clusters is not reproduced for clusters with $r_{\max } \leqslant 3.64 \AA$. For these small clusters the computation yields a broad single peak resembling that experimentally obtained in the mechanically alloyed and milled samples without a sintering treatment. This result indicates that the modification of the shape of the white line can be also accounted as reflecting the short-range order existing in the milled samples and not only as due to a structural phase transformation (cubic-tetra-mono).

Regarding the comparison between the theoretical calculations performed varying the size of the clusters and the experimental XANES an appreciation should be made. It is important to note that while XANES and EXAFS are a short-range techniques, and thus emphasize the order of the next-neighboring coordination around the $\mathrm{Y}$ or $\mathrm{Zr}$ cation, the grain size estimation obtained from XRD is a long-range characteristic length where several unit cells are involved. Our results indicate that these nanocrystalline materials obtained by mechanically alloying milling are single-phase materials that have inherited their local atomic ordering within the first coordination shell. Both XANES and EXAFS spectra show that the local structure in the as-milled samples is highly distorted so that order extends only to the first coordination shells around the absorbing atoms. Upon sintering the compounds evolve toward a long-range ordered lattice and

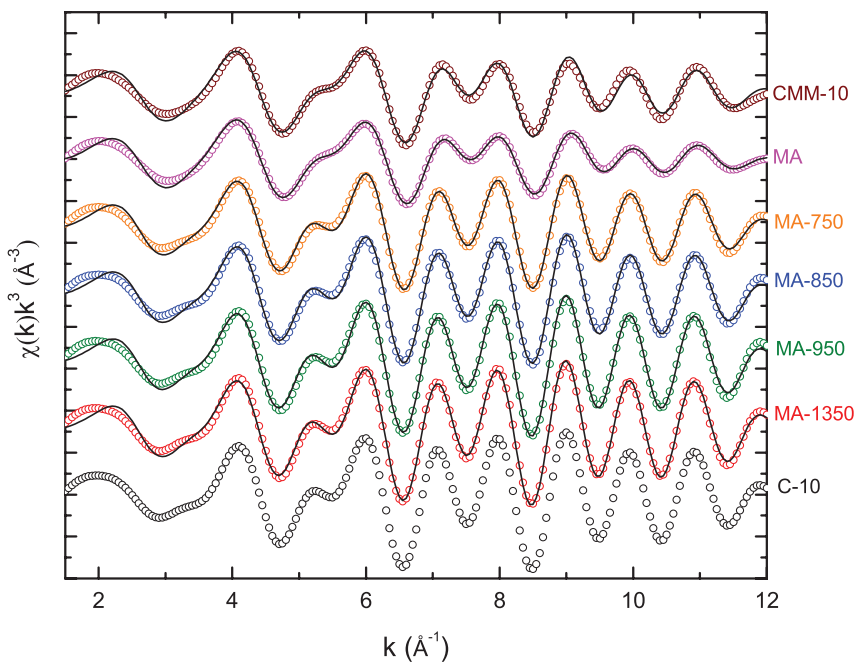

FIG. 8. (Color online) Comparison between the Y $K$-edge back Fourier transform (BFT) of the samples studied in this work. Solid lines represent the fits corresponding to the parameters resumed in Table II. bulklike lattice is already reached. Therefore, we demonstrate here that the observed variations in the XANES profile are exclusively related to the extension of the short-range order existing in the milled samples and not to structural transitions, similarly to the case of as-milled $\mathrm{R}_{2} \mathrm{Ti}_{2} \mathrm{O}_{7}$ pyrochlores. ${ }^{18}$ The exhaustive XANES and EXAFS analysis support that the ionic conductivity associated to the grain boundary should be independent of the grain size. On the other hand, the not-sintered samples exhibit a highly distorted environment where the cation-cation correlation is lost, and consequently the bulk conductivity can be hindered by the disorder.
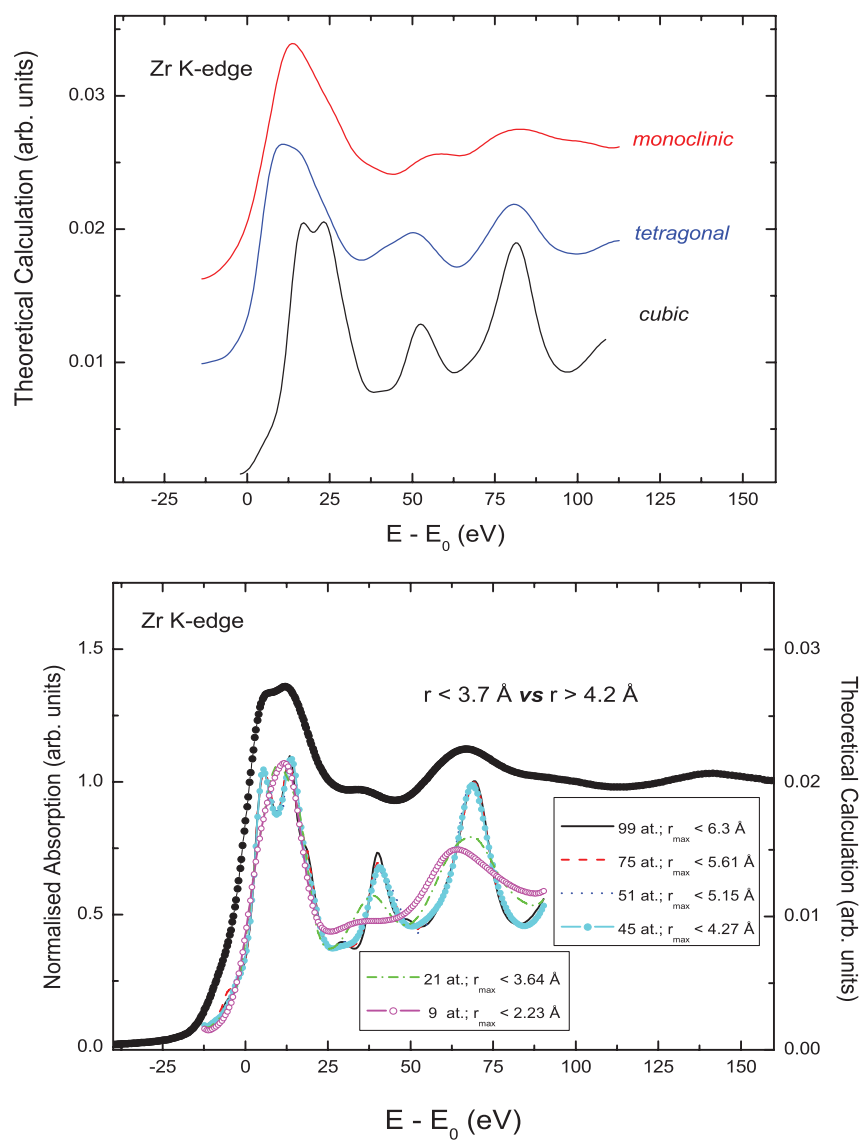

FIG. 9. (Color online) Top: Comparison of the theoretical $\mathrm{Zr}$ $K$-edge XANES spectra calculated for the different zirconia polymorphs. Bottom: Comparison between the experimental $\mathrm{Zr} K$-edge XANES of the cubic C-10 sample and the theoretical calculations performed by varying the size of cluster. 


\section{SUMMARY AND CONCLUSION}

Herein we report a deep x-ray absorption spectroscopy study of nanocrystalline YSZ powders with different order/disorder ratio with the aim of elucidating the previously reported differences in the electrical behavior of grain boundaries and bulk regions. The comparison of the XANES spectra recorded at $\mathrm{Zr}$ and $\mathrm{Y} K$ edge indicates that all sintered MA samples present a very similar spectral shape as for the commercial reference sample, indicating that the local order degree is independent of the grain size. Moreover, the results indicate that although the sample sintered at lowest temperature $\left(750^{\circ} \mathrm{C}\right)$ shows an increased disorder in the second coordination shell the local order is retained. On the contrary, the results obtained for samples mechanically alloyed and milled without further sintering treatment show a highly distorted environment where only the cation-oxygen coordination is defined whereas the cation-cation correlation is lost. In spite of this, the XANES simulations indicate that changes in the as-milled samples are only related to the short-range order and not to a phase transformation. These results are confirmed by the quantitative EXAFS analysis.

\section{ACKNOWLEDGMENTS}

This work was partially supported by Spanish and MAT2011-27573-C04-04 grants, by Junta de Comunidades de Castilla-La Mancha through Project No. PCI-08-0091 and by the Aragón DGA NETOSHIMA grant. The synchrotron radiation experiments were performed at Elettra (Proposal No. 20100420). The assistance of the XAFS beamline staff during the experiments is greatly acknowledged.
*Corresponding author: oscar.juan@uclm.es

${ }^{\dagger}$ Present address: Diamond Light Source Ltd., Harwell Science and Innovation Campus, Didcot, Oxfordshire OX11 ODE, United Kingdom

${ }^{1}$ T. H. Etsell and S. N. Flengas, Chem. Rev. 70, 339 (1970).

${ }^{2}$ B. A. Boukamp, Nature Mater. 2, 294 (2003).

${ }^{3}$ Z. Shao and S. M. Haile, Nature (London) 431, 170 (2004).

${ }^{4}$ J. Maier, Solid State Ionics 175, 7 (2004).

${ }^{5}$ H. L. Tuller, Solid State Ionics 131, 143 (2000).

${ }^{6}$ A. Rivera, J. Santamaria, and C. Leon, Appl. Phys. Lett. 78, 610 (2001).

${ }^{7}$ J. Garcia-Barriocanal, A. Rivera-Calzada, M. Varela, Z. Sefrioui, E. Iborra, C. Leon, S. J. Pennycook, and J. Santamaria, Science 321, 676 (2008).

${ }^{8}$ S. J. Litzelman, J. L. Hertz, W. Jung, and H. Tuller, Fuel Cells 8, 294 (2008).

${ }^{9}$ A. Rivera-Calzada et al., Adv. Mater. 23, 526 (2011).

${ }^{10}$ I. Kosacki, T. Suzuki, V. Petrovsky, and H. U. Anderson, Solid State Ionics 136, 1225 (2000).

${ }^{11}$ P. Mondal, A. Klein, W. Jaegermann, and H. Hahn, Solid State Ionics 136, 1225 (1999).

${ }^{12}$ O. J. Dura, M. A. López de la Torre, L. Vázquez, J. Chaboy, R. Boada, A. Rivera-Calzada, J. Santamaria, and C. Leon, Phys. Rev. B 81, 184301 (2010).

${ }^{13}$ O. J. Durá and M. A. López de la Torre, J. Phys. D: Appl. Phys. 41, 045408 (2008).

${ }^{14}$ A. D. Cicco, G. Aquilanti, M. Minicucci, E. Principi, N. Novello, A. Cognigni, and L. Olivi, J. Phys.: Conf. Ser. 190, 012043 (2009).

${ }^{15}$ D. E. Sayers and B. A. Bunker, X-Ray Absorption (Wiley, New York, 1988), Chap. 6.

${ }^{16}$ B. Ravel and M. Newville, J. Synch. Rad. 12, 537 (2005).

${ }^{17}$ P. H. Citrin, P. Eisenberger, and B. M. Kincaid, Phys. Rev. Lett. 36, 1346 (1976).

${ }^{18}$ M. L. Sanjuán, C. Guglieri, S. Díaz-Moreno, G. Aquilanti, A. F. Fuentes, L. Olivi, and J. Chaboy, Phys. Rev. B 84, 104207 (2011).

${ }^{19}$ K. V. Klementiev, J. Phys. D: Appl. Phys. 34, 209 (2001).

${ }^{20}$ C. R. Natoli and M. Benfatto, (unpublished).
${ }^{21}$ C. R. Natoli and M. Benfatto, J. Phys. (Paris) Colloq. 47, C8 (1986).

${ }^{22}$ M. Benfatto and S. D. Longa, J. Synchrotron Radiat. 8, 1087 (2001).

${ }^{23}$ J. Chaboy and S. Quartieri, Phys. Rev. B 52, 6349 (1995).

${ }^{24}$ J. Chaboy, J. Synchr. Rad. 16, 533 (2009).

${ }^{25}$ J. Chaboy, A. Muñoz-Páez, F. Carrera, P. Merkling, and E. Sánchez Marcos, Phys. Rev. B 71, 134208 (2005).

${ }^{26}$ M. O. Krause and J. H. Oliver, J. Phys. Chem. Ref. Data 8, 329 (1979).

${ }^{27}$ A. V. Chadwick, M. J. Pooley, K. E. Rammutla, S. L. P. Savin, and A. Rougier, J. Phys.: Condens. Matter 15, 431 (2003).

${ }^{28}$ L. M. Acuña, D. G. Lamas, R. O. Fuentes, I. O. Fábregas, M. C. A. Fantini, A. F. Craievich, and R. J. Prado, J. Appl. Crystallogr. 43, 227 (2010).

${ }^{29}$ P. Li, I.-Wei Chen, and J. E. Penner-Hahn, Phys. Rev. B 48, 10074 (1993).

${ }^{30}$ P. Li, I.-Wei Chen, and J. E. Penner-Hahn, Phys. Rev. B 48, 10063 (1993).

${ }^{31}$ D. Michel, E. Gaffet, and P. Berthet, Nanostruct. Mater. 6, 667 (1995).

${ }^{32}$ E. A. Stern, R. W. Siegel, M. Newville, P. G. Sanders, and D. Haskel, Phys. Rev. Lett. 75, 3874 (1995).

${ }^{33}$ S. H. Baker, M. Roy, S. J. Gurman, and C. Binns, J. Phys.: Condens. Matter 21, 183002 (2009).

${ }^{34}$ P. D. Cluskey, R. J. Newport, R. E. Benfield, S. J. Gurman, and G. Schmid, Z. Phys. D 26, S8 (1993).

${ }^{35}$ A. I. Frenkel, C. W. Hills, and R. G. Nuzzo, J. Phys. Chem. B 105, 12689 (2001).

${ }^{36}$ Z. Qi, C. Shi, Y. Wei, Z. Wang, T. Liu, T. Hu, Z. Zhao, and F. Li, J. Phys.: Condens. Matter 13, 11503 (2001).

${ }^{37}$ G. E. Rush, A. V. Chadwick, I. Kosacki, and H. U. Anderson, J. Phys. Chem. B 104, 9597 (2000).

${ }^{38}$ In the case of monoclinic $\mathrm{ZrO}_{2}$ we have considered the average $\mathrm{Zr}-\mathrm{Zr}$ distance reported by Li et al. in Ref. 25.

${ }^{39}$ W. Yuren, L. Kunquan, W. Dazhi, W. Zhonghua, and F. Zhengzhi, J. Phys.: Condens. Matter 6, 633 (1994).

${ }^{40}$ M. Gateshki, V. Petkov, G. Williams, S. K. Pradhan, and Y. Ren, Phys. Rev. B 71, 224107 (2005).

${ }^{41}$ S. Díaz-Moreno, A. Muñoz-Páez, and J. Chaboy, J. Phys. Chem. A 104, 1278 (2000). 
${ }^{42}$ S. Díaz-Moreno and J. Chaboy, J. Phys. Chem. B 113, 3527 (2009).

${ }^{43}$ J. Chaboy and S. Díaz-Moreno, J. Phys. Chem. A 115, 2345 (2011).

${ }^{44}$ J. Chaboy, H. Maruyama, and N. Kawamura, J. Phys.: Condens. Matter 19, 216214 (2007).
${ }^{45}$ J. Chaboy, N. Nakajima, and Y. Tezuka, J. Phys.: Condens. Matter 19, 266206 (2007).

${ }^{46}$ K. Hatada and J. Chaboy, Phys. Rev. B 76, 104411 (2007).

${ }^{47}$ G. Guglieri and J. Chaboy, J. Phys. Chem. C 114, 19629 (2010).

${ }^{48}$ G. Guglieri, E. Céspedes, C. Prieto, and J. Chaboy, J. Phys. Cond. Matter. 23, 206006 (2011). 